\title{
Long-Range Cooperative Disassembly and Aging During Adenovirus Uncoating
}

\author{
Natalia Martín-González@ ${ }^{1, \dagger}$ Pablo Ibáñez-Freire $\odot,{ }^{2, \dagger}$ Álvaro Ortega-Esteban $\odot,{ }^{1}$ Mara Laguna-Castro ${ }^{3, \not}$ \\ Carmen San Martín $\odot,{ }^{3}$ Alejandro Valbuena $\odot,{ }^{4}$ Rafael Delgado-Buscalioni $\odot,{ }^{2,5}$ and Pedro J. de Pablo $\odot^{1,5, *}$ \\ ${ }^{1}$ Department of Condensed Matter Physics, Universidad Autónoma de Madrid, Madrid 28049, Spain \\ ${ }^{2}$ Department of Theoretical Physics of Condensed Matter, Universidad Autónoma de Madrid, \\ Cantoblanco Campus, 28049 Madrid, Spain \\ ${ }^{3}$ Department of Macromolecular Structures, Centro Nacional de Biotecnología (CNB-CSIC), \\ Madrid 28049, Spain \\ ${ }^{4}$ Centro de Biología Molecular "Severo Ochoa," Cantoblanco Campus, 28049 Madrid, Spain \\ ${ }^{5}$ Research Center in Physics of Condensed Matter (IFIMAC), Universidad Autonoma de Madrid, \\ Madrid 28049, Spain
}

(Received 15 October 2020; revised 30 December 2020; accepted 2 February 2021; published 30 April 2021)

\begin{abstract}
Icosahedral virus capsids are closed shells built up with a hexagonal lattice of proteins, which incorporate pentamers at their fivefold vertices. Human adenovirus particles lose pentamers (pentons) during infection under a variety of physicochemical cues, including mechanical pulling of molecular motors and the viscous drag of the cytoplasm. By combining atomic force microscopy experiments with survival analysis and Markovian transition state theory, we investigate the sequence of adenovirus penton disassembly that reveals the aging of the virus structure. We show evidence that the lifetime of pentons gradually decreases, accompanied by capsid softening as neighboring pentons are lost. This cooperative dismantling process, which involves first-neighbor penton-penton distances of at least $45 \mathrm{~nm}$, leads to a $50 \%$ increase in the virus disassembling rate of the virus particle. Theory and experiments fit remarkably well, allowing us to obtain the spontaneous escape rate and the energy barrier of penton disassembly $\left(\sim 30 k_{B} T\right)$. The observed increase in the penton's loss rate reveals long-range structural correlations within the capsid. Our estimations suggest that the mechanical cues arising from the strokes of protein motors carrying the virus to the nucleus could help penton disassembly and warrant the timely delivery of weakenough capsids for adenovirus infection.
\end{abstract}

DOI: 10.1103/PhysRevX.11.021025

\section{INTRODUCTION}

Upon entry into the host cell, eukaryotic viruses undergo a controlled genome uncoating process [1], which usually requires capsid disassembly following a timely precise process. In icosahedral viruses, protein subunits (capsomers) are organized in 20 triangular facets delimited by 12 fivefold vertices forming a structure of hexamers and pentamers [2]. Pentamers are located at each one of the

\footnotetext{
*Corresponding author.

p.j.depablo@uam.es

These authors contributed equally.

${ }^{\star}$ Present address: Department of Molecular Evolution, Center for Astrobiology, CAB(INTA-CSIC), 28850, Torrejón de Ardoz, Spain.

Published by the American Physical Society under the terms of the Creative Commons Attribution 4.0 International license. Further distribution of this work must maintain attribution to the author(s) and the published article's title, journal citation, and DOI.
}

Subject Areas: Biological Physics, Interdisciplinary Physics, Nanophysics
12 vertices surrounded by five hexamers. Therefore, pentons can be understood as the disclinations needed for achieving the curvature of a closed cavity using a planar hexagonal lattice [3]. Because of the geometrical constraints of the pentons within the icosahedral capsid, they are subjected to built-in mechanical stress [4-6]. In fact, some experimental findings report pentons as the weakest capsomers of icosahedral viruses under thermal or chemical stress $[7,8]$. In adenovirus, penton release is a necessary step for uncoating and infectivity [8,9]. Adenoviruses are responsible for acute infections in immunocompromised individuals, but they are also widely used as potential therapeutic tools [10-14]. Human adenovirus type 5 (Ad5) has a pseudo $T=25$ icosahedral shell composed of 12 pentons and 240 hexon trimers occupying the hexamer positions [15]. A protruding fiber is attached to the penton base to mediate binding to the cell membrane protein CAR $[16,17]$ [Fig. 1(a)]. For a successful infection, the adenovirus particle releases protein VI to escape into the cytosol. This process requires capsid opening by penton loss $[18,19]$. Ad5 uncoating has been mimicked in vitro by 
(a)

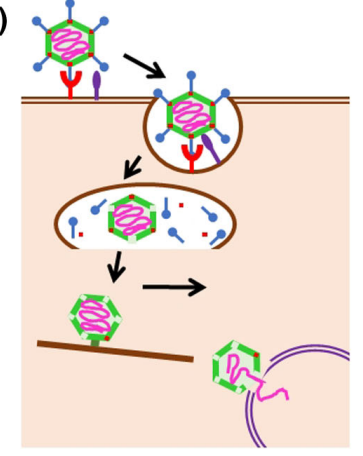

(b)

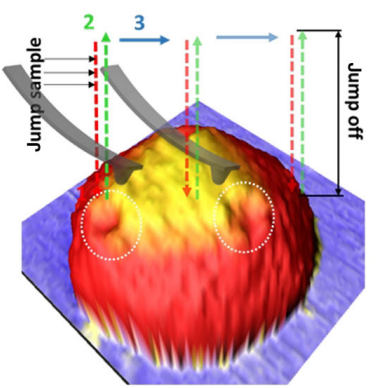

(c)

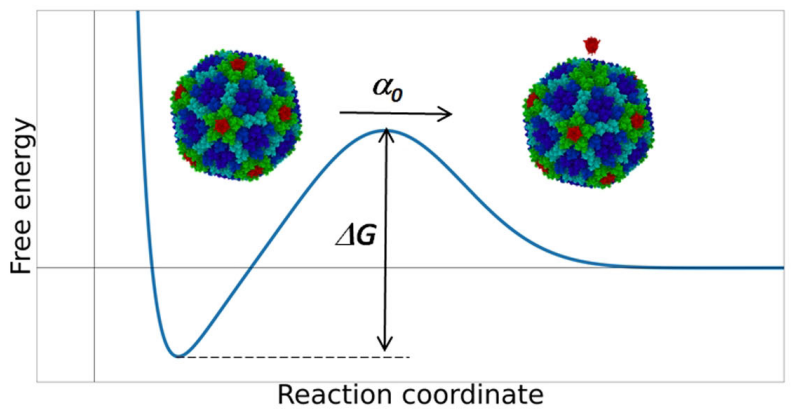

FIG. 1. Adenovirus in biological context, experimental and theoretical setups. (a) Ad5 infection. (b) AFM jumping mode. The tip approaches the sample (1), then withdraws (2), and moves laterally to the next pixel (3). Two missing pentons are shown (white circles). (c) Energetic profile of penton release process when two states are considered.

physicochemical cues such as heat, inducing partial disruption and disassembly of the capsid shell [18]. The cyclic mechanical loading induced by an atomic force microscope (AFM) stylus also supplies energy to the system, allowing it to simultaneously trigger and monitor the disassembly of individual Ad5 particles in real time [19]. Pentons of the facet oriented upwards in mica-adsorbed particles escape sequentially, and afterwards, the shell cracks open, allowing genome release [20]. In this work, we explore the dynamics of Ad5 penton release by combining singleparticle AFM imaging experiments with theoretical modeling based on survival analysis.

\section{METHODS}

\section{A. Production of human adenovirus 5 virus particles}

For this study, we used the E1-deleted Ad5 variant Ad5GL, which lacks fundamental genes for replication but produces structurally wild-type particles in complementing cell lines [21]. Ad5GL was propagated at $37^{\circ} \mathrm{C}$ in HEK 293 cells (MOI of 5) and harvested at $40 \mathrm{~h}$ postinfection. Virus particles were purified by equilibrium centrifugation at $18^{\circ} \mathrm{C}$ in two consecutive $\mathrm{CsCl}$ gradients. Bands extracted from the gradients were desalted on a Bio-Rad 10 DC column and stored in HBS (20 mM HEPES, pH 7.8,
$150 \mathrm{mM} \mathrm{NaCl}$ ) supplemented with $10 \%$ glycerol at $-80^{\circ} \mathrm{C}$ for long-term storage. The virus stock concentration was $5.1 \times 10^{11}$ viral particles $(\mathrm{vp}) / \mathrm{ml}$. For the AFM experiments, samples were thoroughly dialyzed against HBS to remove the storage glycerol and stored in $5 \mu \mathrm{l}$ aliquots at $-20^{\circ} \mathrm{C}$. Samples were diluted four times in HBS supplemented with $5 \mathrm{mM} \mathrm{NiCl}$, for a final concentration of approximately $1.25 \times 10^{10}(\mathrm{vp}) / \mathrm{ml}$, and incubated during $20 \mathrm{~min}$ on cleaved mica. Then, a drop of $500 \mu \mathrm{l}$ of HBS + $5 \mathrm{mM} \mathrm{NiCl} 2$ was placed on the AFM liquid chamber to render a random population of virus particles (Fig. S1, Supplemental Material [22]).

\section{B. AFM experiments}

AFM was used to consecutively image individual Ad5 particles and monitor the evolution of penton loss [19] and to perform benchmark indentation experiments to obtain the spring constants of the viral particles $\left(k_{i}\right)$. In brief, we pipetted a droplet of virus solution on mica in the presence of $\mathrm{NiCl}_{2}$, resulting in a random population of single particles with an intact condition, i.e., with a negligible substrate-induced deformation (Fig. S1). We used rectangular silicon nitride cantilevers RC800PSA (Olympus, Tokyo, Japan) with a nominal spring constant of $0.05 \mathrm{~N} / \mathrm{m}$. This parameter was calculated before each experiment by using Sader's method [23]. The AFM (Nanotec Electrónica S.L., Spain) was operated in Jumping Mode Plus in liquid [Fig. 1(b)] [24,25]. To determine the stiffness of the viral particles, a first image was taken; then, the tip was located at the top of the particle, and a force-versus-distance curve was recorded. During the first stage of indentation, the particles showed a linear deformation from which their spring constant can be derived [26].

\section{Establishing the force range for controlled Ad5 penton release under mechanical perturbations}

AFM imaging at low forces (about $70 \mathrm{pN}$ ) allows us to select individual virus particles adsorbed on the mica surface in a threefold orientation, presenting three intact pentons [27]. For each selected capsid, we acquire a series of AFM images inducing cyclic mechanical stress [19] with forces between 100 and $250 \mathrm{pN}$ [Fig. 2(a), top left]. We note that the applied force should be large enough to remove pentons during one experimental session (about 5 hours) and small enough to avoid sudden uncontrollable large cracks on the viral particles. We find that the lower threshold is $100 \mathrm{pN}$, close to the limit force value, allowing us to obtain AFM images of viruses [24].

The experimental procedure consists of performing a sequence of AFM images of the virus particle using a prefixed imaging force F. Pentons are gradually lost, as more images are taken, neatly showing that pentons are the most fragile structures of the capsid. This result is in agreement with previous experiments where capsids were 
(a)
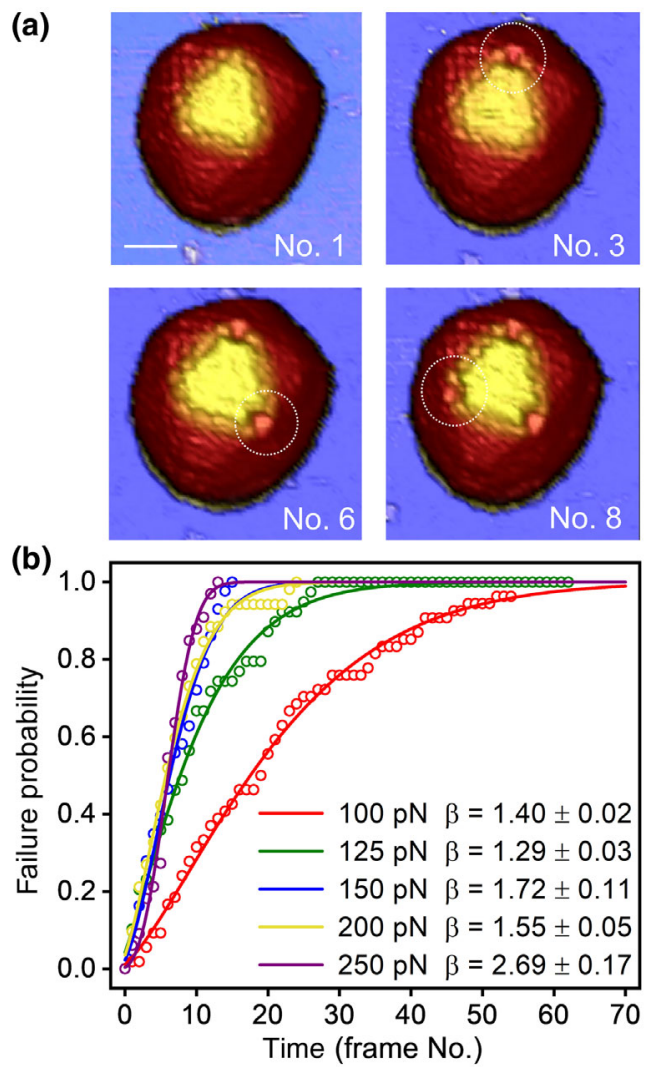

FIG. 2. Penton release analysis by cyclic mechanical stress assay. (a) Individual frames of one Ad5 particle at $150 \mathrm{pN}$ (the scale bar is $37 \mathrm{~nm}$; white circles mark the penton vacancies-see movie in Ref. [22]). (b) Penton release distribution at five forces. Dots represent the experimental data. For example, for $100 \mathrm{pN}$ data (red), about $40 \%$ of the pentons remain on the capsid after 20 frames. Data fitting for a discrete Weibull distribution is shown with solid lines. See details in Fig. S3.

subjected to heat stress $[18,28]$ and with the existence of built-in stress at the vertices [6]. In each experiment, we recorded the image number $\left(N_{i}\right)$ at which we observed the escape of the first $\left(N_{1}\right)$, second $\left(N_{2}\right)$, and third $\left(N_{3}\right)$ pentons [Fig. 2(a)]. Further imaging beyond the dissociation of the third penton revealed how penton-less capsids undergo further disassembly through cracking and crumbling [19] (Fig. S2). In the experiments, we opted to use the applied force $\mathrm{F}$ as the relevant parameter, instead of increasing the imaging rate, which is also known to accelerate capsid disassembly [29]. The imaging rate was fixed to about $10 \mathrm{~ms}$ per pixel $(128 \times 128$ pixels $)$, which is long enough to guarantee the mechanical relaxation of the capsid after obtaining the topographical data of each pixel [30]. This rate includes other relevant experimental parameters, such as the vertical distance traveled by the cantilever at each pixel (jump off) and the sampling rate (jump sample) [Fig. 1(b)] [24]. This rate ensures that each indentation is independent from the previous one or, equivalently, that the capsid does not accumulate any stress over successive
AFM strokes. In mathematical terms, this lack of memory of the previous stroke is called the Markovian hypothesis and will be used (and validated) later within the theoretical framework for the escape process.

\section{ANALYSIS AND RESULTS}

\section{A. Evidence of aging in the probability of penton release: Weibull analysis}

To investigate the statistics of penton escapes, we first collect all the penton failure timepoints (measured in frame number) in the same set of data $\{N\}=\left\{N_{1}, N_{2}, N_{3}\right\}$ irrespective of the chronological order of escape [Fig. 2(b)]. We then construct the histograms for the number of escape events observed at each image $\mathrm{N}$ and integrate (Fig. S3) to obtain the cumulative probability $\mathrm{P}(\mathrm{N})$ for penton rupture [Fig. 2(b)]. $\mathrm{P}(\mathrm{N})$ is thus the probability that some penton is lost after taking $\mathrm{N}$ images at a given force $\mathrm{F}$ or, equivalently, the fraction of released pentons from the total sample at a given frame.

As shown in Fig. 2(b), P(N) significantly deviates from a Poissonian distribution, meaning that the penton failure rate is not constant but instead varies over time (i.e., over the frame number $\mathrm{N})$. To get more insight, we fit $\mathrm{P}(\mathrm{N})$ with a Weibull distribution [31,32], which, in a survival analysis, is a standard way to model processes with a time-dependent failure rate. As the variable $\mathrm{N}$ is an integer, we use the discrete version of the Weibull distribution whose cumulative probability is

$$
P(N)=1-\exp \left[-\left((N+1) /\left\langle N_{p}\right\rangle\right)^{\beta}\right] .
$$

Here, $\left\langle N_{p}\right\rangle$ represents the average lifetime of a penton in terms of the number of images. Note that the failure rate varies with $\mathrm{N}$ as $[d(\log (1-P(N))) / d N]=\left(\beta /\left\langle N_{p}\right\rangle\right)$. $\left(N /\left\langle N_{p}\right\rangle\right)^{\beta-1}$, being determined by $\left\langle N_{p}\right\rangle$ and the so-called shape parameter $\beta$. Importantly, $\beta$ determines how the failure rate varies over time. Specifically, $\beta=1$ corresponds to a constant failure rate, and in this case, the Weibull distribution becomes equivalent to a Poissonian distribution. By contrast, $\beta>1$ is indicative of aging: The failure rate increases over time (i.e., with $\mathrm{N}$ ). Experimental penton-escape probabilities [Fig. 2(b)] are found to be in excellent agreement with aging, i.e., with Weibull distributions having $\beta>1$. In particular, we find $\beta \approx 1.5$ for the range $100 \mathrm{pN}<\mathrm{F}<200 \mathrm{pN}$, while for $F=250 \mathrm{pN}$, it increases up to $\beta \approx 2.5$, which indicates fast aging. In fact, the increase in failure rate with time is even faster than predicted by the Weibull test, as we did not consider that the probability of finding a survival penton decreases after another penton(s) disappears. For instance, after one of the three accessible pentons is lost, the probability of hitting one of the remaining pentons decreases by a factor $2 / 3$, and this contributes to a spurious decrease in the Weibull analysis of the failure rate. As an aside, during the AFM 
experiments, we observe that when applying forces higher than $150 \mathrm{pN}$, the experimental probability curves start to overlap [Fig. 2(b)], preventing the use of higher forces. As shown in the Supplemental Material [22], this experimental resolution limit in force agrees with our theoretical prediction for having a probability higher than $95 \%$ of penton release during the acquisition of the first image (Fig. S4).

The increase of the penton release rate along the experiment is a landmark of aging, which might arise either from an accumulation of capsid stress during each pixel acquisition or from the fact that we did not take into account the chronological order of penton escape in the analysis. It is important to elucidate between both causes because the first one (stress accumulation) would be against the Markovian hypothesis. To analyze this point, we remove the memory stored in the chronological chain of escape events by resetting the time counter each time an escape event takes place. This approach allows us to study each penton-escape event independently. To this end, for each applied force, we gather three types of "time-interval" data: $\left\{n_{1}\right\}=\left\{N_{1}\right\}$, $\left\{n_{2}\right\}=\left\{N_{2}-N_{1}\right\}$, and $\left\{n_{3}\right\}=\left\{N_{3}-N_{2}\right\}$, which correspond to the survival times for the first, second, and third pentons, respectively. Notably, Weibull tests based on $n_{i}$, with $i=\{1,2,3\}$, revealed that the resulting probabilities $P_{i}(n)$ correspond to exponentials (i.e., $\beta \approx 1.0$, see Table S1). The escape of a single penton is thus a Poissonian (memoryless) process and confirms the validity of the "Markovian hypothesis"; i.e., the tapping process does not introduce stress accumulation in the capsid, as the particle has enough time to relax between successive pixels (taken every $10 \mathrm{~ms}$ ). This result is to be expected, as the mechanical relaxation time of virus capsids measured from vibrational modes [33] is about 100 ps.

We then estimate the average escape rates for the first, second, and third pentons, which correspond to welldefined, "relaxed" states of the capsid. Interestingly, we find that after the first penton is released, the average lifetime of the following pentons is reduced. For instance, at $\mathrm{F}=100 \mathrm{pN}$, the average lifetime of the first penton is about $37.5 \mathrm{~min}$ (12.5 images), while, for the second and third pentons, it is about 27 min (9 and 8 images, respectively) (Table S2). This fact explains the increase in failure rate over image time observed in the Weibull analysis. The result indicates that pentamer disassembly events constitute a cooperative stepwise process, where the first step facilitates the following ones. Cooperativity has a sense of "purpose," which could be attributed to the need of the capsid to gradually dismantle to achieve successful infection.

\section{B. Estimation of the spontaneous penton-escape rate}

We now focus on an analytical determination of the probability of releasing the $i$ th penton after taking $n$ images $P_{i}(n)(i=1,2,3)$, which is amenable for comparison with experiments. From a theoretical point of view, the transition kinetics of penton release can be simplified as a two-state process (bound and unbound) [Fig. 1(c)]. In the absence of external mechanical perturbations (i.e., zero external work, $W=0$ ), the transition rate $\alpha_{0}$ for a penton to change from bound to unbound in a thermal bath is related to the free energy well $\Delta \mathrm{G}$ by a simple Arrhenius-type law,

$$
\alpha_{0}=\omega_{0} \exp \left(\frac{-\Delta \mathrm{G}}{k_{B} T}\right)
$$

Note that $\alpha_{0}$ is the spontaneous or natural escape rate, and $\omega_{0}$ is the natural frequency of fluctuations of the penton in the energy well. To extract $\alpha_{0}$ and then estimate $\Delta \mathrm{G}$, we use a well-known strategy in transition theory [34], which consists in applying mechanical work $\mathrm{W}$ into the system to lower the free energy well $(\Delta \mathrm{G}-\mathrm{W})$ and accelerating the pentonescaping events to gather statistics in reasonable experimental times. In the jumping mode imaging of the AFM, the tip induces mechanical work to the capsid through an intermittent force $f_{\mathrm{AFM}}(t)$ whose time dependence is similar to a tunable top-hat function (see the Appendix and Fig. S5). From the virus perspective, each stroke of this long series of indentations is extremely slow, and during each single jump, the applied work $W(t)>0$ increases the transition rate to the unbonded state according to [34]

$$
\alpha(t)=\alpha_{0} \cdot e^{W(t) / k_{B} T},
$$

where $k_{B}$ is the Boltzmann constant and T is the temperature. To take a step further, we first propose a linear relation between the work applied by the AFM tip and the set point force (maximum F), $W=-F x_{\mathrm{ef}}$, where $x_{\mathrm{ef}}$ is a constant effective "indentation length." A second route consists of using the harmonic-well approximation, $F=-k x$, with an effective spring constant of the capsid, $k$. The indentation is then $x=(-F / k)$, and the applied work becomes quadratic in the applied force, $W=\left(\frac{1}{2}\right)\left(F^{2} / k\right)$, allowing us to compare theoretically estimated values of spring constants $k$ with the experiments. The free parameters of the two-state model are $\alpha_{0}$ and either $x_{\text {ef }}$ or the effective spring constant $k$, which will be calculated by fitting the theoretical $P_{i}(n)$ with the experimental results.

The derivation of the single-penton-escape probability $P_{i}(n)$ is detailed in the Appendix. Starting from the expression for $\alpha(t)$ in Eq. (3), we first calculate the probability $P_{f}(t)$ of penton failure after a given time, during a pixel. We are working with a Markov unidirectional process (from a bound to an unbound state) representing a first-order kinetic reaction. In an ensemble average, the increase in the number of unbound (or free) pentons, $d P_{f}(t)$, would be simply proportional to the population of bonded pentons, and the master equation reads 

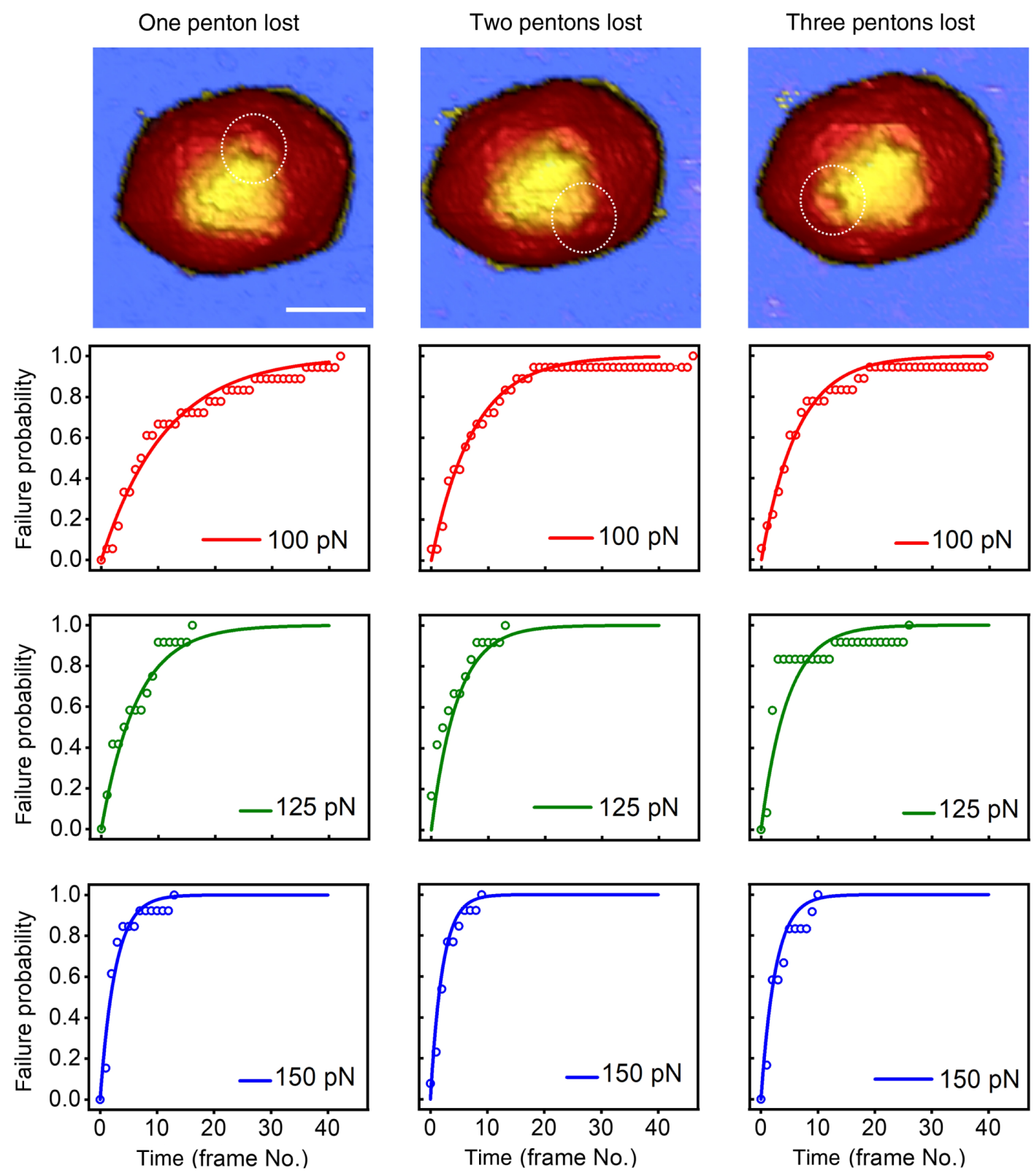

FIG. 3. Penton release distribution under Markov process model. Top: AFM images taken after the first, second, and third pentonescape events (in the left panel, the scale bar corresponds to $44 \mathrm{~nm}$ ). White circles mark the penton vacancies. Bottom: cumulative probability of penton failure $P_{i}(n)$ for successive penton escapes when the capsid is exposed to strokes of forces of $100 \mathrm{pN}, 125 \mathrm{pN}$, and $150 \mathrm{pN}$ (right panel). Dots represent the experimental data, and dashed lines represent the model results [Eq. (5)].

$$
d P_{f}(t)=\alpha(t)\left[1-P_{f}(t)\right] d t .
$$

The applied force $f_{\mathrm{AFM}}(t)$ used in Eq. (3) is experimentally known [19] (see the Appendix), which permits us to perform a change of variable, mapping the image number " $n$ " with real time in seconds. This mapping is essential as Eq. (2) requires one to have the natural $\alpha_{0}$ escape rate in proper inverse-of-time units, to estimate the energy barrier $\Delta \mathrm{G}$. Briefly, by integrating Eq. (4), we first determine the escape probability after a single pixel and then integrate over one complete image and finally over an arbitrary number $n_{i}$ of images. The resulting expression for the probability of releasing the $i$ th penton is

$$
P_{i}\left(n_{i}\right)=1-\left(1-C_{i}\right)^{n_{i}},
$$

where $C_{i}$, given in the Appendix, depends on the applied force $\mathrm{F}$ and the free parameters we seek to find $\left(x_{\mathrm{ef}}, k_{i}\right.$, and the natural escape rate of the $i$ th penton, $\left.\alpha_{0, i}\right)$. By fitting the theoretical probability $P_{i}(n)$ [Eq. (5) and the Appendix] with the experimental results, we estimate $x_{\mathrm{ef}}$ and $\alpha_{0, i}$. The value of $x_{\mathrm{ef}}$ is found to be independent of the penton index, and the best fit $\left(x_{\mathrm{ef}}=0.18 \pm 0.03 \mathrm{~nm}\right)$ is used for all the penton sequences. As shown in Fig. 3, the theoretical model is in excellent agreement with experimental data. Additionally, we estimate an increase in the spontaneous escape rate as the penton-release order " $i$ " 
increases: $\alpha_{0,1}=(2.7 \pm 0.1) \times 10^{-4} \mathrm{~s}^{-1}, \alpha_{0,2}=(4.7 \pm 0.2) \times$ $10^{-4} \mathrm{~s}^{-1}$, and $\alpha_{0,3}=(6.4 \pm 0.3) \times 10^{-4} \mathrm{~s}^{-1}$ (first, second, and third pentons, respectively). Interestingly, the spontaneous escape rate of the third penton is about twice as fast as the first one. As a curiosity, these values of the spontaneous rates are of the same order as those reported in the mechanical unfolding of proteins [35-37].

\section{Weakening of the capsid stiffness}

It has been reported that penton vacancies may be accompanied by a change in the global capsid stiffness [38,39]. Although this might seem logical, it is not a common feature in nanomaterials [40]. The increase of the failure rate that we observe after the release of the first penton indicates a global effect, which could be ascribed to the capsid structure softening. In addition, this capsid softening is not obvious, as pentons are the weakest capsomers of the virus structure $[7,8]$. We can estimate the decrease in stiffness by using the ansatz $W=\left(\frac{1}{2}\right)\left(F^{2} / k\right)$, based on the energy of a harmonic well with effective local stiffness $k$. The form of the theoretical failure probabilities was found to be in good agreement with the experimental trends (Fig. S6) and allowed us to fit the theoretical $P_{i}(n)$ with the experimental outcome to obtain estimations for the capsid stiffness. For intact capsids, we found $k_{0}=0.50 \pm 0.02 \mathrm{~N} / \mathrm{m}$, while for capsids without one or two pentons, we obtained $k_{1}=0.42 \pm 0.01 \mathrm{~N} / \mathrm{m}$ and $k_{2}=0.41 \pm 0.01 \mathrm{~N} / \mathrm{m}$, respectively. These values were compared with independent experimental data for intact capsids $\left(k_{0}\right)$ and a group of 18 pentonless capsids $\left(k_{i}\right)$. In these benchmark experiments, Ad5 pentonless particles were obtained by applying a temperature stress ( $T=40^{\circ} \mathrm{C}$ during 5 minutes in solution), which caused viral particles to lose at least one penton [18]. The benchmark experiments yielded $k_{0}=0.58 \pm 0.01 \mathrm{~N} / \mathrm{m}$ (for a capsid population of $N=50$ ) [20] for intact particles and $0.42 \pm 0.06 \mathrm{~N} / \mathrm{m}(N=18)$ [41] for heated capsids. These values are consistent with those obtained from escape probability distributions, where the penton number and release order were considered. The similarity between the estimated values of $k_{i}$ is remarkable if one considers that both experiments are radically different. The benchmark experiments measured $k$ from the linear regime of the forceindentation curves, using single indentation with a force of a few $\mathrm{nN}$, which ultimately causes large capsid deformations of tens of nm, as well as cracks and capsid breaking. Here, we typically apply about $10^{5}$ gentle AFM strokes of about $100 \mathrm{pN}$, at low frequency $(10 \mathrm{~ms})$.

\section{Estimation of the free energy barrier for penton release}

We have seen that losing pentons affects the Ad5 particle in two ways: (i) a gradual increase in the penton-escape rate and (ii) a decrease in the capsid stiffness. From the ratio $\alpha_{0,3} / \alpha_{0,1}$, Eq. (2) allows us to assess the difference between the energy barriers of the third and first pentons, which is about $\Delta G_{3}-\Delta G_{1}=-1 \mathrm{k}_{B} T$. This value might indicate a small but noticeable modification in the molecular configuration of the residues sustaining the penton (after two partners are lost), which leads to the particle softening.

Our analysis also allows us to estimate the average energy barrier for the penton release, $\Delta \mathrm{G}$. For the process, we need to estimate $\omega_{0}$, the rate of exploration of the local well of the pentamer. A reasonable estimate is based on the balance of cohesion forces and environmental friction, and it yields $\omega_{0}=(k / \xi) \approx 10^{10} \mathrm{~Hz}$ (the penton's friction coefficient $\xi$ is taken as the Stokes value in water, where the dynamic viscosity is $\eta \sim 10^{-3} \mathrm{Pas}$ ). Alternative estimations of $\omega_{0}$ require the cutoff radius of the well $\lambda$ (a subnanometric length of the order of the junction between the penton and the neighboring hexamers). We infer that $\lambda$ must be similar to the displacement produced by the limit force at which the loss of the penton can be seen as extremely fast (i.e., approximately within a single image $)$, i.e., $\lambda=\left(F_{\text {limit }} / k\right) \simeq 0.5 \mathrm{~nm}$. Thermal fluctuations lead to an average kinetic energy $M v^{2}=k_{B} T$, and using $\omega_{0}=(v / \lambda)$, this leads to $\omega_{0}=6.4 \times 10^{9} \mathrm{~Hz}$. Vibrations around the quadratic potential lead to $\omega_{0}=$ $(k / M)^{1 / 2} /(2 \pi)=10^{10} \mathrm{~Hz}$. These three estimations are quite close, and we conclude that $\omega_{0} \approx 7.0 \times 10^{9} \mathrm{~Hz}$, which is in good agreement with the relaxation rates of capsid units evaluated from NMR spectra [42] and from the vibrational modes via light scattering [33]. Taking logarithms in Eq. (2) and averaging over $\left\{\alpha_{0, i}\right\}$, we predict an energy barrier of $\Delta \mathrm{G} \approx(30 \pm 1) k_{B} T$. This value is consistent with those reported in the literature for capsid assembly, either from coarse-grained simulations [4] or calorimetry studies [43]. The pairwise interaction between capsid units is known to be between 5 and $6 k_{B} T$. For the five contacts of the pentamer, this interaction leads to a total binding free energy of about $30 k_{B} T$, which agrees with our result.

\section{DISCUSSION}

Theoretical simulations of virus assembly consider capsomer interactions to first neighborhoods $[4,44]$. In principle, this type of building method might suggest that penton disruption is a local event depending solely on the neighboring capsomers, i.e., hexons. However, we measure a significant increase of the penton-escape rate after the first penton is lost. In the Ad5 capsid, neighboring pentons are about $45 \mathrm{~nm}$ away, so the loss of one penton is not expected to induce a strong local modification of the protein environment surrounding the other pentons. Since every penton is surrounded by similar hexons, the penton loss rate will not depend on the chronological order as long as their local structural environment remains unchanged. However, our analysis clearly shows that, far from being a local event, penton disassembly depends on the global state of the 
capsid. Additionally, our analysis confirms a measurable softening of Ad5 capsids after one penton is lost. Both results suggest that the cooperativity in the penton-release process (aging) is related to a gradual modification in the global mechanical structure of the capsid. Notably, $45 \mathrm{~nm}$ would be the largest correlation distance identified between structural elements in a virus capsid [45] and other protein assemblies [46-49]. Further support of this conclusion is given in theoretical studies, which reveal that the tangential stress distribution along the capsid presents long-range correlations connecting pentons, being particularly strong in $T=25$ capsids [6]. Aside from a global change in the particle stiffness, some enhancement in fluctuations and particle breathing, perhaps also influenced by loss of internal components such as protein VI or core protein $\mathrm{V}$ [18], might also facilitate the next penton escape.

We now explore the implications of our findings in the timeline of adenovirus uncoating [9]. In the initial stage, integrins bind to the penton base and induce the release of the associated penton. Penton loss facilitates externalization of protein VI, whose lytic activity disrupts the endosome membrane to allow virus escape at $15 \mathrm{~min}$ post infection (p.i.). Afterwards, the semidisrupted particle is pulled along the microtubules, against the drag of the viscous cytoplasm, by both dynein (minus-end) and kinesin (plus-end) motor proteins, which bind to the capsid hexons [50,51]. During this randomized travel (active diffusion) along the microtubule network, the Ad5 capsid keeps losing capsomers [50,52]. Each stroke of the molecular motor introduces mechanical work $\left(\sim 10 k_{B} T\right.$ [53]) into the capsid at a rate of about $10 \mathrm{~Hz}$ and corresponds to a step of about $5 \mathrm{~nm}$. During travel to the nucleus that covers between 5 and $10 \mu \mathrm{m}$ [51], the partially disassembled capsids are subjected to thousands of these strokes. This energy is partly dissipated to the motor-protein linker and to the surrounding area, but part of this mechanical energy $(W)$ will reach the capsid, thus increasing the pentonescape rate by the Boltzmann factor $e^{W / k_{B} T}$. We find that the penton lifetimes exponentially decay with the applied force, at least for $100 \mathrm{pN} \leq 200 \mathrm{pN}$. As an educated guess, if $W \sim 2.5 k_{B} T$ (a quarter of the protein-motor power stroke), the lifetime of a penton would be reduced by a factor 10. Once at the nuclear membrane (45-60 min p.i.), the virus particle is broken by the coupled action of the attached kinesin motor pushing in opposite directions [51]. At this stage, most virus particles appear to be weak enough to release the viral genome into the nuclear pore. The work introduced by each tap over the long series $\left(\sim 10^{5}\right)$ of gentle AFM strokes mimics the mechanical queues that the virion receives during travel to the nucleus.

Our in vitro experiments reveal that genome ejection takes place after at least three pentons have been removed [Fig. 4(a)]. Figure 4(b) shows that the dsDNA escapes from the virus cage through big cracks that require the additional disassembly of hexons [19]. In vivo, semidisrupted particles (a)
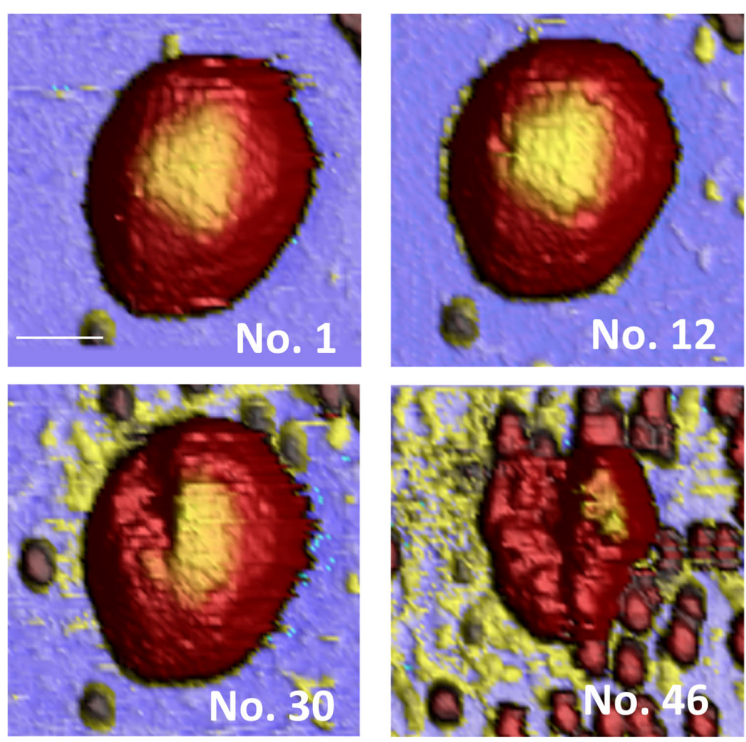

(b)
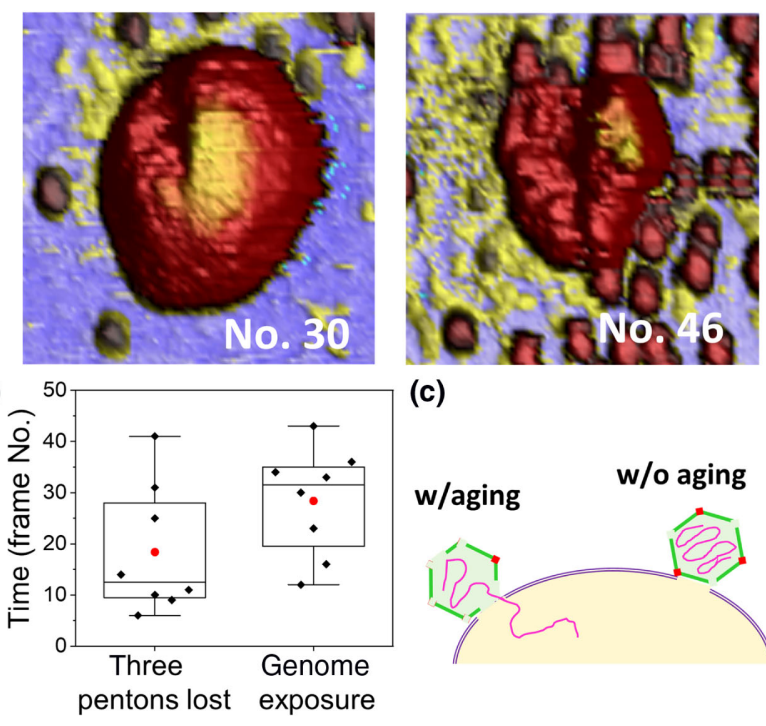

(c)

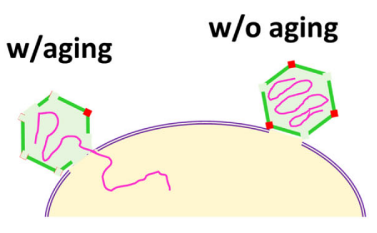

FIG. 4. Genome exposure and cooperative penton release. (a) Top-left panel: intact virus particle (scale bar corresponds to $40 \mathrm{~nm}$ ). Top-right panel: the same particle with three penton vacancies at frame No. 12. Bottom-left panel: the same particle at frame No. 30, showing some DNA released. Bottom-right panel: the final state of the collapsed particle. (b) Chart presenting the number of frames required for releasing the pentons and inducing DNA exposure. (c) Model of viral-impaired uncoating at the nuclear pore assuming aging (left) and nonaging (right) penton release.

must be strong enough to preserve genome integrity when traveling from the endosome to the nucleus [54]. Most likely, the uncoating process is adapted to achieve these two apparently contradictory goals: (i) to keep the capsid stable enough during the transport to the nucleus and (ii) for the capsid to become weak enough once it arrives at the nuclear pore. Retaining a large number of pentons would guarantee a safe trip to the nucleus, but it might delay or even preclude the final uncoating at the nuclear pore [Fig. 4(c)]. The survival analysis presented here estimates the average natural lifetime of the first penton in about $1 / \alpha_{0,1} \sim 1 \mathrm{~h}$. The lifetime is gradually reduced to $1 / \alpha_{0,2} \sim 30 \mathrm{~min}$ (second penton) and $1 / \alpha_{0,3} \sim 20 \mathrm{~min}$ (third penton). The whole process is over $50 \%$ faster than a noncooperative penton-escaping sequence. We conclude that the cooperative penton disassembly helps in achieving the required capsid aging on time for the genome to be successfully delivered at the nuclear pore (i.e., between 
60 and 90 min p.i.). In view of the relatively adjusted times in the infectious cycle, a 50\% delay might already prevent the virus from releasing its dsDNA on time (e.g., by allowing detection by cell defense mechanisms or ending up stuck on the nuclear pore) [Fig. 4(c)]. Therefore, we suggest that the increased disruption rate of the pentons helps fine-tune the uncoating process of human adenovirus.

\section{ACKNOWLEDGMENTS}

Work at the C.S.M. laboratory is supported by the Spanish Agencia Estatal de Investigación and European Regional Development Fund (BFU2016-74868-P and PID2019-104098 GB-I00/AEI/10.13039/501100011033); the Ministerio de Economía y Competitividad of Spain (BFU2013-41249-P and BIO2015-68990-REDT); and the Agencia Estatal CSIC (2019AEP045). The CNB-CSIC is further supported by a Severo Ochoa Excellence grant (SEV 2017-0712). M. L.-C. was a recipient of a Youth Employment Initiative contract co-funded by the European Social Fund and Regional Madrid Government (PEJ16/ MED/TL-0935). P. J.d.P. acknowledges support by grants from the Spanish Ministry of Economy, Industry and Competitiveness projects (FIS2017- 89549-R; "Maria de Maeztu" Program for Units of Excellence in R\&D MDM2014-0377; and FIS2017-90701- REDT) and the Human Frontiers Science Program (HFSPO RGP0012/2018). R.D.-B. acknowledges funding from Spanish Ministry of Econonomy, Industry and Competitiveness FIS201786007-C3-1-P and the IFIMAC local project R\&D MDM-2014-0377.

A. V., P. J.d. P., and R. D. B. designed the research; M. L.-C. conducted specimen production; N. M.-G., A. O.-E., A. V., and P. J. d. P. conducted AFM experiments; A. V., P. I. F., and R. D. B. conducted theoretical analysis; C. S. M. supervised specimen production; R. D. B, A. V., and P.J.d.P. wrote the paper, with input from all other authors.

The authors declare no competing interest.

\section{APPENDIX: PROBABILISTIC MARKOVIAN MODEL DESCRIPTION}

We derive a theoretical model for the statistics of the lag time between successive penton-escape events, treated as a Markovian process (see main text). The experimental results (Fig. 3 and Table S1) indicate that these events corresponds to Poissonian processes with a well-defined, time-independent escape rate. We separately consider the survival times for the first, second, and third pentons, taking the survival "time" (in image units) as the difference between successive escape events. In order to reproduce the experimental data, the theoretical model should reconstruct each step of the experimental procedure, which is composed of three parts: first, an individual pixel (or indentation); second, a single image with $M=128 \times 128$ pixels (this corresponds to the experimental resolution); and third, a number " $n$ " of images for the escape event to occur. We derive the probability of losing a penton over each of these steps, to finally obtain $P(n)$ in Eq. (5).

To begin, one needs to consider the spatial details of a single indentation, which has two possible outcomes: (i) The indentation touches an area that "stimulates" a penton, or (ii) it touches a region that disturbs the penton. In the first case, we consider that a certain work $W$ is applied to the penton, while in the latter case, $W=0$. The small indentation area, about $(5 \mathrm{~nm})^{2}(\mathrm{SM})$, permits us to assume that indentation has a local effect; i.e., we consider $W=0$ if the tip touches a region of the capsid where the penton has already been lost. Therefore, the case $W=0$ includes two subgroups: indentations outside the capsid (on the substrate) and indentations in a region of the capsid that has already lost its penton. By inspecting many experimental images, we calculate the average fraction of the image covered by the capsid, which is noted as the parameter $\nu$. Within the capsid domain, the fraction of the image that corresponds to the "region of influence" of an exposed penton is noted as $\rho_{i} \nu$, where $\rho_{i}$ is the fraction of the remaining exposed pentons. Since there are three pentons exposed to the AFM tip [Fig. 2(a), this parameter will take the values $\rho_{1}=1$ when no penton has been lost, $\rho_{2}=2 / 3$ when one penton has left the virus, and, finally, $\rho_{3}=1 / 3$ when only one penton remains. Let $P^{\text {out }}$ be the probability of losing a penton if the indentation does not "touch" the penton $(W=0)$, and $P^{\text {in }}$ the probability of losing a penton after "touching" it with the AFM tip $(W>0)$. The total probability of losing the "ith" penton after resolving one pixel is then

$$
B_{i}=(1-\nu) P^{\text {out }}+\nu\left(\rho_{i} P^{\text {in }}+\left(1-\rho_{i}\right) P^{\text {out }}\right) .
$$

We now deduce the form of $P^{\text {out }}$ and $P^{\text {in }}$ and integrate over pixels and images to derive Eq. (5), i.e., the probability of losing the $i$ th penton after a number $n$ of images, $P(n)$. This value is precisely the quantity amenable for comparison with experiments.

We start by considering a single indentation. Equation (4) is the master equation for the probability $P_{f}(t)$ of pentons being in the "free" (unbonded) state at time t. This equation is equivalent to a first-order kinetic reaction and requires one to evaluate the transition rate $\alpha(t)$. To this end, we need to consider the location of the indentation. The case "indentation out" corresponds to the AFM tip not perturbing the penton (either because the AFM tip touches the substrate or avoids it in the capsid without some penton). In such a case ( $W=0$ ), we set $\alpha(t)=\alpha_{0}$, which is the spontaneous escape rate, related to the free energy barrier $\Delta \mathrm{G}$ by the Kramer relation given in Eq. (2),

$$
\alpha(t)=\alpha_{0}=\omega_{0} \exp \left[-\Delta \mathrm{G} / k_{B} T\right] \quad \text { (indentation "out"). }
$$

Integrating the master equation, Eq. (4), leads to the probability of escaping after a single indentation that is "outside" the penton domain, 


$$
P^{\text {out }}=1-\exp \left(-\alpha_{0} \mathrm{~T}\right)
$$

where $\mathrm{T}$ denotes the time required for one indentation (10 ms).

Second, if the indentation stimulates the penton domain (we denote this case as "in"), the tip introduces some mechanical work, which increases the escape rate as described in Eq. (3),

$$
\begin{aligned}
\alpha(t) & =\omega_{0} \exp \left[-(\Delta \mathrm{G}-W(t)) / k_{B} T\right] \\
& =\alpha_{0} \exp \left[W(t) / k_{B} T\right] \quad \text { (indentation "in"). }
\end{aligned}
$$

The "in" scenario requires a more detailed analysis. Integration of the master equation, Eq. (4), using Eq. (A3) leads to

$$
\begin{aligned}
P_{f}^{\text {in }}(t) & =1-\exp \left(-\int_{0}^{t} \alpha(t) d t^{\prime}\right) \\
& =1-\exp \left(\alpha_{0} \int_{0}^{t} \exp \left(W\left(t^{\prime}\right) / k_{B} T\right) d t^{\prime}\right) .
\end{aligned}
$$

To proceed with the integration of the exponent, we first examine the profile of the indentation force as a function of time, $f_{\mathrm{AFM}}(t)$. This profile, measured experimentally, has the form of a top-hat function with five different steps. In the initial step $\left(t_{0}<t<t_{1}\right.$, where $\left.t_{0}=0\right)$, the tip approaches the sample and the applied force is zero; this "free-force" step has a duration $t_{1}=t_{F}$. Upon contact $\left(t_{1}<t<t_{2}\right)$, the force increases linearly in time until it reaches the maximum (set point) force $F$. The elapsed time during this "ramp" step is $t_{R}$. For $t_{2}<t<t_{3}$, the force $F$ is held fixed over a "contact" time $t_{C}$, and the rest of the process follows similarly in inverse order: force ramp back to zero $\left(t_{3}<t<t_{4}\right)$ and tip moving away from the sample $\left(t_{4}<t<t_{5}\right)$. The total time needed for a single indentation is thus $\mathrm{T}=t_{5}=2 t_{F}+2 t_{R}+t_{C}$, with $t_{1}=t_{F}$, $t_{2}=t_{1}+t_{R}, t_{3}=t_{2}+t_{C}, t_{4}=t_{3}+t_{R}$. As a function of time, the applied force $f_{\mathrm{AFM}}(t)$ is a piecewise function (Fig. $\quad$ S5): $\quad f_{\mathrm{AFM}}(t)=0 \quad$ for $\quad t_{0}<t<t_{1} ; \quad f_{\mathrm{AFM}}(t)=$ $F\left(t-t_{F}\right) / t_{R}$ for $t_{1}<t<t_{2} ; f_{\mathrm{AFM}}(t)=F$ for $t_{2}<t<t_{3}$; $f_{\mathrm{AFM}}(t)=-F\left(t-t_{F}-2 t_{R}-t_{C}\right) / t_{R}$ for $t_{3}<t<t_{4}$; and $f_{\mathrm{AFM}}(t)=0$ for $t_{4}<t<t_{5}$. The duration of each step depends, in turn, on the maximum force $F$ and can be determined experimentally,

$$
\begin{aligned}
t_{F} & =0.00588-0.00000078 F \\
t_{R} & =0.00000088-0.00000041 F \\
t_{C} & =0.00076-0.000000077 F
\end{aligned}
$$

The next piece of information requires some relation between the applied work and the force. The first model is based on $W=-F r$ (where $r=x_{\text {ef }}$ in the main text is a constant effective "indentation length"). We first perform the integrals $I_{k}$ appearing in the exponents of Eq. (A4) for each one of the five time intervals $t_{k-1}<t<t_{k}$ of the indentation process (we define $\beta=1 / k_{B} T$ ),

$$
\begin{aligned}
I_{1}\left(t_{0}, t_{1}\right)= & I_{5}\left(t_{0}, t_{1}\right)=\int_{t_{0}}^{t_{1}} \exp (0) d t=\left(t_{1}-t_{0}\right) \\
I_{2}\left(t_{0}, t_{1}\right)= & \int_{t_{0}}^{t_{1}} \exp \left(\frac{t-t_{F}}{t_{R}} \beta F r\right) d t \\
= & \frac{t_{R}}{\beta F r}\left(\exp \left[\beta F r\left(\frac{t_{1}-t_{F}}{t_{R}}\right)\right]\right. \\
& \left.-\exp \left[\beta F r\left(\frac{t_{0}-t_{F}}{t_{R}}\right)\right]\right) \\
I_{3}\left(t_{0}, t_{1}\right)= & \left.t_{1}-t_{0}\right) \exp (\beta F r) \\
I_{4}\left(t_{0}, t_{1}\right)= & \int_{t_{0}}^{t_{1}} \exp \left(-\frac{t-2 t_{F}-2 t_{R}-t_{C}}{t_{R}} \beta F r\right) d t \\
= & -\frac{t_{R}}{\beta F r}\left(\exp \left[-\beta F r\left(\frac{t_{1}-2 t_{F}-2 t_{R}-t_{C}}{t_{R}}\right)\right]\right. \\
& \left.-\exp \left[-\beta F r\left(\frac{t_{0}-2 t_{F}-2 t_{R}-t_{C}}{t_{R}}\right)\right]\right) \quad(\mathrm{A} 6)
\end{aligned}
$$

Using these integrals, the probability of encountering a free penton over time results in another piecewise function, such that $P^{\text {in }}(\mathrm{T})=1-\exp \left[-\alpha_{0}\left(I_{1}\left(t_{0}, t_{1}\right)+I_{2}\left(t_{1}, t_{2}\right)+\right.\right.$ $\left.\left.I_{3}\left(t_{2}, t_{3}\right)+I_{3}\left(t_{3}, t_{4}\right)+I_{5}\left(t_{4}, \mathrm{~T}\right)\right)\right]$, where we recall that $\mathrm{T}=2 t_{F}+2 t_{R}+t_{C}$ corresponds to the total time lapse of a single indentation cycle $(\mathrm{T}=10 \mathrm{~ms})$. After some algebra, this yields the probability of losing a penton in a single indentation performed "in" a pentamer domain,

$$
\begin{aligned}
P^{\text {in }} \equiv & P^{\text {in }}(T)=1-\exp \left[-\alpha_{0}\left(\exp (\beta F r) t_{C}\right.\right. \\
& \left.\left.+\left(\frac{1}{\beta F r}\right)(\exp (\beta F r)-1) 2 t_{R}+2 t_{F}\right)\right] .
\end{aligned}
$$

In the second model, the work is expressed as $W(t)=\left(\frac{1}{2}\right) F^{2} / k$, and a similar procedure leads to

$$
\begin{aligned}
P^{\text {in }} \equiv & P^{\text {in }}(T)=1-\exp \left[-\alpha_{0}\left(\exp \left(\frac{\beta F^{2}}{2 k}\right) t_{C}\right.\right. \\
& \left.\left.+\sqrt{\frac{2 \pi k}{\beta F^{2}}} \operatorname{erfi}\left(\frac{\beta F^{2}}{2 k}\right) t_{R}+2 t_{F}\right)\right] .
\end{aligned}
$$

Following Eq. (A1), collecting the spatial details of the indentation (AFM tip either "in" or "out") leads to the total probability for penton unbinding during a single indentation,

$$
B_{i}=(1-\nu) P^{\text {out }}+\nu\left(\rho_{i} P^{\text {in }}+\left(1-\rho_{i}\right) P^{\text {out }}\right),
$$

where $\rho_{i}=(4-i) / 3$ is the fraction of remaining pentons in the capsid area facing the AFM, with $i=1$ 
(intact capsid), $i=2$ (one penton is lost), and $i=3$ (two pentons are lost).

We can follow a similar procedure to model the image acquisition process. In this case, we face a discrete case since we are interested in the escape probability as a function of the number of pixels we are indenting (unlike the previous case, in which we were interested in the evolution of the probability as a function of time along a single indentation). In this case, we introduce the stochastic transition matrix of the process,

$$
\mathbf{T}=\left(\begin{array}{cc}
1-B & B \\
0 & 1
\end{array}\right)
$$

The evolution of the discrete process can be written in terms of the transition matrix as

$$
\left[\begin{array}{ll}
P_{\text {bonded }}^{p+1} & P_{\text {free }}^{p+1}
\end{array}\right]=\left[\begin{array}{ll}
P_{\text {bonded }}^{p} & P_{\text {free }}^{p}
\end{array}\right]\left(\begin{array}{cc}
1-B & B \\
0 & 1
\end{array}\right),
$$

i.e., according to a Markovian process, the state after the $p+1$ indentation only depends on the state at the previous pixelation $p$. If the initial state is $\left[P_{\text {bonded }}^{0}, P_{\text {free }}^{0}\right]=[1,0]$, the probability after performing $m$ indentations is thus obtained by the recursive application of the transition matrix, resulting in $\left(P_{b}^{m}, P_{f}^{m}\right)=\left(P_{b}^{0}, P_{f}^{0}\right) \mathbf{T}^{m}$. One can compute the $m$ power of the stochastic matrix, $\mathbf{T}^{m}$, as

$$
\left(\begin{array}{cc}
1-B & B \\
0 & 1
\end{array}\right)^{m}=\left(\begin{array}{cc}
(1-B)^{m} & 1-(1-B)^{m} \\
0 & 0
\end{array}\right)
$$

so the probability of losing a penton after $m$ indentations is $p(m)=\mathrm{P}_{f}^{m}=1-(1-B)^{m}$. In deriving this expression, we assume that the pixels are taken at random locations, which is not strictly true because during a single image, the pixelation follows a well-defined order. However, this point is not crucial because we are interested in the probability of losing a penton after passing all the $\mathrm{M}$ pixels of the image. The large number of pixels per image $(M=128 \times 128)$ indicates that the above expression is appropriate. Taking everything into account, we obtain the probability of losing a penton after taking one image (i.e., M pixels),

$$
C:=p(M)=1-(1-B)^{M} .
$$

Moreover, $\mathrm{M}$ is a large number, so this expression can be approximated by $C:=1-(1-B)^{M} \simeq 1-\exp (-B M)$, which is easier to work with. In conclusion, the probability of releasing the $i$ th pentamer after a single image is

$$
C_{i}:=1-\exp \left(-B_{i} M\right)
$$

where $B_{i}$ is given in Eq. (A1) and involves Eq. (A4), (A7), or (A8).
The last step is to compute $P_{i}\left(n_{i}\right)$, the release probability after a number $n_{i}$ of images (where we recall $n_{i}$ starts to be counted since the previous penton "i-1" is lost). We can compare this information with experiments. The situation is formally analogous to the previous case, and following a similar approach, we arrive at Eq. (5) (main text). Note that $C_{i}$ is given in Eq. (A9), and $P_{i}\left(n_{i}\right)$ is compared with experimental results in Fig. 3.

[1] S. J. Flint, V. R. Racaniello, G. F. Rall, A. M. Skalka, and L. W. Enquist, Principles of Virology (Wiley, 2015), https://www.wiley.com/en-us/Principles+of+Virology\%2C +Volume+1\%3A+Molecular+Biology $\% 2 \mathrm{C}+5$ th+Edition-p9781683673606.

[2] D. L. Caspar and A. Klug, Physical Principles in the Construction of Regular Viruses, Cold Spring Harbor Symp. Quant. Biol. 27, 1 (1962).

[3] W. S. Klug, R. F. Bruinsma, J. P. Michel, C. M. Knobler, I. L. Ivanovska, C. F. Schmidt, and G. J. L. Wuite, Failure of Viral Shells, Phys. Rev. Lett. 97, 228101 (2006).

[4] R. Zandi, D. Reguera, R. F. Bruinsma, W. M. Gelbart, and J. Rudnick, Origin of Icosahedral Symmetry in Viruses, Proc. Natl. Acad. Sci. U.S.A. 101, 15556 (2004).

[5] W. S. Klug, W. H. Roos, and G. J. L. Wuite, Unlocking Internal Prestress from Protein Nanoshells, Phys. Rev. Lett. 109, 168104 (2012).

[6] R. Zandi and D. Reguera, Mechanical Properties of Viral Capsids, Phys. Rev. E 72, 021917 (2005).

[7] D. Patterson, B. Schwarz, K. El-Boubbou, J. Oost, P. Prevelige, and T. Douglas, Virus-like Particle Nanoreactors: Programmed Encapsulation of the Thermostable CelB Glycosidase inside the P22 Capsid, Soft Matter 8, 10158 (2012).

[8] U.F. Greber and J.W. Flatt, Adenovirus Entry: From Infection to Immunity, Annu. Rev. Virol. 6, 177 (2019).

[9] U. F. Greber, M. Willetts, P. Webster, and A. Helenius, Stepwise Dismantling of Adenovirus 2 during Entry into Cells, Cell 75, 477 (1993).

[10] J. W. Shiver, T.-M. Fu, D. C. Kaslow, and E. A. Emini, Replication-Incompetent Adenoviral Vaccine Vector Elicits Effective Anti-Immunodeficiency-Virus Immunity, Nature (London) 415, 331 (2002).

[11] M. A. F. V. Gonçalves and A. A. F. De Vries, Adenovirus: From Foe to Friend, Rev. Med. Virol. 16, 167 (2006).

[12] M. O. Lasaro and H. C. J. Ertl, New Insights on Adenovirus as Vaccine Vectors, Mol. Ther. 17, 1333 (2009).

[13] M. Yamamoto and D. T. Curiel, Current Issues and Future Directions of Oncolytic Adenoviruses, Mol. Ther. 18, 243 (2010).

[14] M. A. Kay, State-of-the-Art Gene-Based Therapies: The Road Ahead, Nat. Rev. Genet. 12, 316 (2011).

[15] X. Dai, L. Wu, R. Sun, and Z. H. Zhou, Atomic Structures of Minor Proteins VI and VII in Human Adenovirus, J. Virol. 91, e00850-17(2017).

[16] M. Y. Nakano, K. Boucke, M. Suomalainen, R. P. Stidwill, and U.F. Greber, The First Step of Adenovirus Type 2 Disassembly Occurs at the Cell Surface, Independently of 
Endocytosis and Escape to the Cytosol, J. Virol. 74, 7085 (2000).

[17] C. J. Burckhardt, Drifting Motions of the Adenovirus Receptor CAR and Immobile Integrins Initiate Virus Uncoating and Membrane Lytic Protein Exposure, Cell Host and Microbe 10, 105 (2011).

[18] A. J. Pérez-Berná, A. Ortega-Esteban, R. MenéndezConejero, D. C. Winkler, M. Menéndez, A. C. Steven, S. J. Flint, P. J. De Pablo, and C. San Martín, The Role of Capsid Maturation on Adenovirus Priming for Sequential Uncoating, J. Biol. Chem. 287, 31582 (2012).

[19] A. Ortega-Esteban, A. J. Pérez-Berná, R. MenéndezConejero, S. J. Flint, C. San Martín, and P. J. de Pablo, Monitoring Dynamics of Human Adenovirus Disassembly Induced by Mechanical Fatigue, Sci. Rep. 3, 1434 (2013).

[20] N. Martín-González, M. Hernando-Pérez, G. N. Condezo, M. Pérez-Illana, A. Šiber, D. Reguera, P. Ostapchuk, P. Hearing, C. San Martín, and P. J. de Pablo, Adenovirus Major Core Protein Condenses DNA in Clusters and Bundles, Modulating Genome Release and Capsid Internal Pressure, Nucleic Acids Res. 47, 9231 (2019).

[21] T. Seki, I. Dmitriev, E. Kashentseva, K. Takayama, M. Rots, K. Suzuki, and D. T. Curiel, Artificial Extension of the Adenovirus Fiber Shaft Inhibits Infectivity in Coxsackievirus and Adenovirus Receptor-Positive Cell Lines, J. Virol. 76, 1100 (2002).

[22] See Supplemental Material at http://link.aps.org/ supplemental/10.1103/PhysRevX.11.021025 for extra data and figures.

[23] J. E. Sader, J. W. M. Chon, and P. Mulvaney, Calibration of Rectangular Atomic Force Microscope Cantilevers, Rev. Sci. Instrum. 70, 3967 (1999).

[24] A. Ortega-Esteban, I. Horcas, M. Hernando-Pérez, P. Ares, A. J. Pérez-Berná, C. San Martín, J. L. Carrascosa, P. J. de Pablo, and J. Gómez-Herrero, Minimizing Tip-Sample Forces in Jumping Mode Atomic Force Microscopy in Liquid, Ultramicroscopy 114, 56 (2012).

[25] I. Horcas, R. Fernández, J. M. Gómez-Rodríguez, J. Colchero, J. Gómez-Herrero, and A. M. Baro, WSXM: A Software for Scanning Probe Microscopy and a Tool for Nanotechnology, Rev. Sci. Instrum. 78 (2007).

[26] M. Jiménez-Zaragoza, M. P. L. Yubero, E. Martín-Forero, J. R. Castón, D. Reguera, D. Luque, P. J. de Pablo, and J. M. Rodríguez, Biophysical Properties of Single Rotavirus Particles Account for the Functions of Protein Shells in a Multilayered Virus, eLife 7, 1 (2018).

[27] C. Zeng, M. Hernando-Pérez, B. Dragnea, X. Ma, P. Van Der Schoot, and R. Zandi, Contact Mechanics of a Small Icosahedral Virus, Phys. Rev. Lett. 119, 038102 (2017).

[28] M. Benevento, S. Di Palma, J. Snijder, C. L. Moyer, V. S. Reddy, G. R. Nemerow, and A. J. R. Heck, Adenovirus Composition, Proteolysis, and Disassembly Studied by in-Depth Qualitative and Quantitative Proteomics, J. Biol. Chem. 289, 11421 (2014).

[29] A. Valbuena and M. G. Mateu, Kinetics of Surface-Driven Self-Assembly and Fatigue-Induced Disassembly of a VirusBased Nanocoating, Biophys. J. 112, 663 (2017).

[30] A. Arkhipov, W. H. Roos, G. J. L. Wuite, and K. Schulten, Elucidating the Mechanism behind Irreversible Deformation of Viral Capsids, Biophys. J. 97, 2061 (2009).
[31] B. W. Weibull, A Statistical Distribution Function of Wide Applicability, J. Appl. Mech. 18, 293 (1951), http://web.cecs .pdx.edu/cgshirl/Documents/Weibull-ASME-Paper-1951 .pdf.

[32] T. Nakagawa and S. Osaki, The Discrete Weibull Distribution, IEEE Trans. Reliab. R-24, 300 (1975).

[33] B. Stephanidis, S. Adichtchev, P. Gouet, A. McPherson, and A. Mermet, Elastic Properties of Viruses, Biophys. J. 93, 1354 (2007).

[34] G. Bell, Models for the Specific Adhesion of Cells to Cells, Science 200, 618 (1978).

[35] M. Carrion-Vazquez, A. F. Oberhauser, S. B. Fowler, P. E. Marszalek, S. E. Broedel, J. Clarke, and J. M. Fernandez, Mechanical and Chemical Unfolding of a Single Protein: A Comparison, Proc. Natl. Acad. Sci. U.S.A. 96, 3694 (1999).

[36] A. Valbuena, J. Oroz, R. Hervas, A. M. Vera, D. Rodriguez, M. Menendez, J. I. Sulkowska, M. Cieplak, and M. CarrionVazquez, On the Remarkable Mechanostability of Scaffoldins and the Mechanical Clamp Motif, Proc. Natl. Acad. Sci. U.S.A. 106, 13791 (2009).

[37] J. Stigler and M. Rief, Ligand-Induced Changes of the Apparent Transition-State Position in Mechanical Protein Unfolding, Biophys. J. 109, 365 (2015).

[38] A. Ortega-Esteban, G. N. Condezo, A. J. Pérez-Berná, M. Chillón, S. J. Flint, D. Reguera, C. San Martín, and P. J. De Pablo, Mechanics of Viral Chromatin Reveals the Pressurization of Human Adenovirus, ACS Nano 9, 10826 (2015).

[39] W. H. Roos, K. Radtke, E. Kniesmeijer, H. Geertsema, B. Sodeik, and G. J. L. Wuite, Scaffold Expulsion and Genome Packaging Trigger Stabilization of Herpes Simplex Virus Capsids, Proc. Natl. Acad. Sci. U.S.A. 106, 9673 (2009).

[40] G. López-Polín, C. Gómez-Navarro, V. Parente, F. Guinea, M. I. Katsnelson, F. Pérez-Murano, and J. Gómez-Herrero, Increasing the Elastic Modulus of Graphene by Controlled Defect Creation, Nat. Phys. 11, 26 (2015).

[41] A. Ortega-Esteban, Doctorate thesis (2015), https:// repositorio.uam.es/handle/10486/670035.

[42] C. D. Anobom, S. C. Albuquerque, F. P. Albernaz, A. C. Oliveira, J. L. Silva, D. S. Peabody, A. P. Valente, and F. C. L. Almeida, Structural Studies of MS2 Bacteriophage Virus Particle Disassembly by Nuclear Magnetic Resonance Relaxation Measurements, Biophys. J. 84, 3894 (2003).

[43] S. Katen and A. Zlotnick, The Thermodynamics of Virus Capsid Assembly, Methods Enzymol. 455, 395 (2009).

[44] J. D. Perlmutter, C. Qiao, and M. F. Hagan, Viral Genome Structures Are Optimal for Capsid Assembly, eLife 2, e00632 (2013).

[45] A. Roy and C. B. Post, Long-Distance Correlations of Rhinovirus Capsid Dynamics Contribute to Uncoating and Antiviral Activity, Proc. Natl. Acad. Sci. U.S.A. 109, 5271 (2012).

[46] D. J. E. Callaway and Z. Bu, Nanoscale Protein Domain Motion and Long-Range Allostery in Signaling Proteins-A View from Neutron Spin Echo Spectroscopy, Biophys. Rev. Lett. 7, 165 (2015).

[47] J. Li, D. J. E. Callaway, and Z. Bu, Ezrin Induces LongRange Interdomain Allostery in the Scaffolding Protein NHERF1, J. Mol. Biol. 392, 166 (2009). 
[48] T. Domínguez-Gil, M. Lee, I. Acebrón-Avalos, K. V. Mahasenan, D. Hesek, D. A. Dik, B. Byun, E. Lastochkin, J. F. Fisher, S. Mobashery, and J. A. Hermoso, Activation by Allostery in Cell-Wall Remodeling by a Modular Membrane-Bound Lytic Transglycosylase from Pseudomonas Aeruginosa, Structure 24, 1729 (2016).

[49] M. R. Williams, S. J. Lehman, J. C. Tardiff, and S. D. Schwartz, Atomic Resolution Probe for Allostery in the Regulatory Thin Filament, Proc. Natl. Acad. Sci. U.S.A. 113, 3257 (2016).

[50] K. H. Bremner, J. Scherer, J. Yi, M. Vershinin, S. P. Gross, and R. B. Vallee, Adenovirus Transport via Direct Interaction of Cytoplasmic Dynein with the Viral Capsid Hexon Subunit, Cell Host Microbe 6, 523 (2009).

[51] J. Zhou, J. Scherer, J. Yi, and R. B. Vallee, Role of Kinesins in Directed Adenovirus Transport and Cytoplasmic Exploration, PLoS Pathogens 14, e1007055 (2018).
[52] A. Rentsendorj, J. Xie, M. MacVeigh, H. Agadjanian, S. Bass, D.-H. Kim, J. Rossi, S. F. Hamm-Alvarez, and L. K. Medina-Kauwe, Typical and Atypical Trafficking Pathways of Ad5 Penton Base Recombinant Protein: Implications for Gene Transfer, Gene Therapy 13, 821 (2006).

[53] J. Howard, Mechanics of Motor Proteins and the Cytoskeleton Sunderland (Oxford University Press, 2001), https://global.oup.com/ushe/product/mechanics-of-motorproteins-and-the-cytoskeleton-9780878933334?cc=es $\langle=e n \&$.

[54] S. Strunze, M. F. Engelke, I.-H. Wang, D. Puntener, K. Boucke, S. Schleich, M. Way, P. Schoenenberger, C. J. Burckhardt, and U.F. Greber, Kinesin-1-Mediated Capsid Disassembly and Disruption of the Nuclear Pore Complex Promote Virus Infection, Cell Host Microbe 10, 210 (2011). 